\title{
Influence of age on pain sensitivity in response to paw pressure and formalin injection in rats: A role of nitric oxide
}

\author{
Nisreen M. Omar ${ }^{1}$, Abdelaziz M. Hussein ${ }^{1}$, Hala Abdel Malek ${ }^{1}$, Mohamed Ahdy A. A. Saad ${ }^{1}$ \\ and Dalia M. Saleh ${ }^{2}$ \\ ${ }^{1}$ Department of Physiology and Clinical Pharmacology, Faculty of Medicine, Mansoura University, Mansoura, Egypt \\ ${ }^{2}$ Department of Anatomy and Embryology, Faculty of Medicine, Mansoura University, Mansoura, Egypt
}

\begin{abstract}
The effect of age on pain response to paw pressure and intraplantar formalin injection in rats is elucidated. Pain responses evoked by mechanical pressure on hind paw and intraplantar injection of formaldehyde (5\%) into the hind paw were evaluated in groups of adult, young and aged male Sprague Dawley rats, after intraperitoneal (i.p.) or intracerebroventricular (i.c.v.) injection of L-arginine or NG-nitro-L-arginine methyl ester (L-NAME). Nicotinamide adenine dinucleotide phosphate (NADPH)-diaphorase staining was done in the two groups. The results show that pain response was reduced in the aged rats and enhanced pain response to paw pressure in aged rats only. L-arginine (i.c.v.) had no effect on pain response to paw pressure in the two groups but enhanced biphasic pain response to formalin. L-NAME (i.p. and i.c.v.) suppressed pain response to paw pressure in the two groups. L-NAME (i.c.v.) suppressed pain response to formalin during the acute phase and enhanced it during the late phase. NADPH-diaphorase activity was significantly greater in young rats. In conclusion, pain response is blunted in the aged rats. NO might be involved in mechanical nociception in aged rats and in formalin-induced nociception in both groups. NO blockade has an antinociceptive effect on pain response. Central NO has dual role in pain response evoked by formalin.
\end{abstract}

Key words: Nitric oxide - Pain sensitivity - Young and aged rats - Paw pressure - Formalin

\section{Introduction}

Pain is defined as an unpleasant sensory and emotional experience associated with actual or potential tissue damage (Freire et al. 2009). Nociceptive sensitization (hyperalgesia) that results from a persistent nerve or tissue damage is perceived as a decreased threshold to noxious stimuli with the value of protecting the injured site and avoiding further damage (Wall and Woolf 1984). The effect of aging on pain sensitivity and threshold is not well defined (Harkins et al. 1994). Some animals studies have indicated that reactivity to pain decreased in 25 month-old rats compared to 3 month-old animals either in response to thermal and mechanical stimuli (Akunne and Soliman

Correspondence to: Hala Abdel Malek, Department of Physiology and Clinical Pharmacology, Faculty of Medicine, Mansoura University, Mansoura, Egypt

E-mail: halamalek@live.com
1994) or to thermal and electrical stimuli (Crosby et al. 2006). On the other hand, Islam et al. (1993) reported an enhanced nociception in 12, 18 and 24 month-old rats compared to 6 month-old rats; while Crisp et al. (1994) have shown that pain reactivity is enhanced in 25 monthold rats when compared to 15 month-old rats but not to 6 month-old rats. Meanwhile, Goicoechea et al. (1997), using the tail-shock test, found no difference in nociception between 25 and 10 month-old rats.

The mechanism of nociceptive sensitization is widely studied and has been attributed to peripheral and central pathways. Peripheral sensitization is shown to be mediated by inflammatory mediators such as prostaglandin E2 (Taiwo and Levine 1989; England et al. 1996), bradykinin, nerve growth factor, and nitric oxide (NO) in the injured site (Milligan and Watkins 2009). These mediators increase the sensitivity and excitability of primary afferent nociceptors. On the other hand, there is still much debate about the mediators involved in central sensitization but there is 
accumulating evidence of the important role of NO (Hopper and Garthwaite 2006; Haghikia et al. 2007). NO is synthesized from L-arginine by nitric oxide synthase (NOS) enzymes (Moncada 1994). Of the three identified NOS isoenzymes, the neuronal NOS (nNOS) has been associated with nitrergic signalling in the brain. However, many evidences have shown that endothelial NOS (eNOS) also contributes to the physiological actions of NO in the brain (Garthwaite 2008). As indicated above, there is accumulating evidence that NO has a role in nociception. However, there is still controversy whether NO has a pronociceptive or antinociceptive role. Thus, some studies have shown that treatment with inhibitors of NOS prevents the development of hyperalgesia in rat in response to either thermal or mechanical noxious stimuli, suggesting an important role of NO in nociception (Moore et al. 1991; Handy and Moore 1998). Further, intracutaneous or intravenous injection of NO solution has been found to evoke pain in humans, indicating a role of NO in peripheral nociception (Holthusen and Arndt 1994, 1995). By contrast, several studies have demonstrated that stimulation of the arginine-NO-cGMP pathway exerts antinociceptive effect (Duarte and Ferreira 1992, 2000; Brito et al. 2006). Further, some studies have demonstrated that the antinociceptive properties of some drugs are mediated through NO. Pretreatment with NOS inhibitors has been shown to interfere with the effects of these drugs, including clonidine (Przesmycki et al. 1999), morphine (Pataki and Telegdy 1998; Abacioğlu et al. 2001), D-Pen2,5-enkephalin (Chen and Pan 2003), sildenafil (Patil et al. 2004), and muscarinic agonists in the rostral ventral medulla of rats (Iwamoto and Marion 1994).

Thus, the main aim of the present study was to further elucidate the effect of age on pain sensitivity in rats in response to thermal and mechanical stimuli, and also to investigate the importance of NO in peripheral and central nociceptive responses related to the age.

\section{Materials and Methods}

\section{Experimental animals}

Sprague-Dawley male rats were kept in the animal care facility at Physiology Department, Mansoura Faculty of Medicine under a 12-h light/dark cycle and had free access to food and water. All experimental protocols were approved by Mansoura Faculty of Medicine Animal Care and Use Committee.

\section{Experimental design}

Rats were divided into 2 main groups: young adult rats ( 2 month-old, $n=12$, body weight $=180-200 \mathrm{~g}$ ) and aged rats ( 24 month-old, $n=12$, body weight $=350-380 \mathrm{~g}$ ). Two groups were subjected to pain sensitivity test in response to chemical and mechanical stimuli to compare the effect of age on nociception. Then, to further evaluate the role of $\mathrm{NO}$ in nociception in the aged rats, NG-nitro-L-arginine methyl ester (L-NAME, NOS inhibitor) and L-arginine (NO precursor) were injected intraperitoneal (i.p.) and intracerebroventricular (i.c.v.). According to the method of administration of the agent, rats were further divided into 2 subgroups:

1. L-NAME pretreated group $(n=6)$, which was further subdivided into 2 subgroups: a) received i.p. L-NAME at a dose of $50 \mathrm{mg} / \mathrm{kg}, 30 \mathrm{~min}$ before testing (Gholipour et al. 2008); b) received i.c.v. L-NAME at a dose of $1.0 \mu \mathrm{g}$, 15 min before testing (Hashemi et al. 2010).

2. L-arginine pretreated group $(n=6)$, which was further subdivided into 2 subgroups: a) received i.p. L-arginine at a dose of $200 \mathrm{mg} / \mathrm{kg}, 45 \mathrm{~min}$ before testing (Gholipour et al. 2008); b) received i.c.v. L-arginine at a dose of $1.0 \mu \mathrm{g}, 15 \mathrm{~min}$ before testing (Hashemi et al. 2010).

\section{Drugs}

The drugs used in this study were L-arginine and L-NAME (Sigma fine chemical Co., USA). The drugs were prepared freshly on the day of the experiment by dissolving in sterile $0.9 \% \mathrm{NaCl}$ solution.

\section{Surgical and stereotaxic procedures}

A mixture of diazepam $(5 \mathrm{mg} / \mathrm{kg})$ and ketamine $\mathrm{HCl}$ (75 mg/kg) was used to anesthetize the experimental animals. Then, the rat was fixed into stereotaxis table (Stoelting stereotaxic unit, model 51600, USA) and a short incision was made along the midline of the scalp using a scalpel. The skin was pulled back to expose the calvarium. A small hole was made on the calvarium, at stereotaxic coordinates: $0.95 \mathrm{~mm}$ posterior to bregma, $1.4 \mathrm{~mm}$ lateral to the midline. Then, a stainless steel needle of outer diameter $0.5 \mathrm{~mm}$ was fixed at this point, by dental cement to a depth $3.2 \mathrm{~mm}$ below the dorsal surface of the brain (Kutlu et al. 2003). Rats were allowed to recover their activities for 10 days in separate cages before pain threshold testing procedure.

\section{Central microinjection procedure}

Injection (i.c.v.) was made using the microinjection technique of Haley and McCormick (1957), using a 10- $\mu$ l microsyringe (Hamilton, Reno, NV, USA). A volume of $5 \mu \mathrm{l}$ (containing $1 \mu \mathrm{g}$ ) of drug solution was delivered directly into the lateral cerebral ventricle over $30 \mathrm{~s}$. 


\section{Pain threshold testing}

Mechanical stimulus: paw withdrawal threshold (PWT). The paw pressure test consisted of gently holding the body of the rat while the hind paw was exposed to controlled increasing mechanical pressure. The analgesy-meter (Ugo-Basile, Varese, Italy, cat. \# 7200) is designed to exert a force on the paw that increases at a constant rate, similar to the Randall and Sellito (1957) test of mechanical nociception. Force was applied to the right hind paw that was placed under a small plinth under a cone-shaped plunger with a rounded tip. The operator depressed a pedal-switch to start the mechanism that exerted force. The force in grams at which the rat withdrew its paw was defined as the paw pressure threshold. The baseline paw pressure was measured before injecting vehicle or drug. Five determinations per animal (with interval between sequential measurements of about 10-15 min) were collected in each test session, averaged and expressed in grams.

Formalin test: chemical threshold. The formalin test was performed as previously described (Jourdan et al. 2000). In brief, the rat was acclimated to the observation chamber (transparent glass box permitting an unobstructed view of rat's paws to the observer) for $\sim 20 \mathrm{~min}$. Then, the rat was restrained and $10 \mu \mathrm{l}$ of formaldehyde (5\%) was injected subcutaneously into the right hind paw using a Hamilton microsyringe with a $30 \mathrm{G}$ needle and replaced again in the observation chamber. Then, the duration (in seconds), the animal spent licking, lifting and/or favoring the injected paw was recorded between 0-5 min (early phase) and 15-60 min (late phase) after the formalin injection.

\section{NADPH-diaphorase technique}

In order to identify the effect of aging on the neuronal NOS distribution in the rat brain and spinal cord, nicotinamide adenine dinucleotide phosphate (NADPH)-diaphorase technique was used.

Specimens' collection and sectioning. Animals were anaesthetized with ether and perfused intracardially with 200-300 $\mathrm{ml}$ of $10 \%$ neutral buffered formalin after a brief rinse of the vasculature with $0.9 \%$ saline. An incision was made through abdomen along the length of the diaphragm. A first cut through the connective tissue at the bottom of diaphragm was made to allow access to rib cage. A second cut was made through ribs and the heart was exposed. A needle was inserted into the left ventricle and secured in its entry site. The valve of the perfusion pump was released and fluid was allowed to flow in a slow, steady way of around $20 \mathrm{ml} / \mathrm{min}$. A cut was made in the right atrium with sharp scissors to allow the solution to flow freely. After completion of the process of fixation the brains and the lumbosacral region of the spinal cords were carefully dissected out. The brains were sliced into small pieces by removing the anterior part of the forebrain by a coronal section at the rostral end of corpus callosum. The hind brain was separated by a transverse section at the lower end of the midbrain. The tissue was then stored in the same fixative for 4-5 days in order to provide an adequate NADPH-diaphorase staining. After being fixed, the tissues were stored in $15 \%$ sucrose in $0.1 \mathrm{M}$ phosphate buffer, $\mathrm{pH} 7.4$, overnight at $4^{\circ} \mathrm{C}$ to minimize the formation of ice crystals. The tissues were sectioned with a cryostat at 8 and $40 \mu \mathrm{m}$ thick and collected on coated slides and stained with gallocyanin chrom alum stain for Nissle substance and with nitro-blue tetrazolium for NADPH-diaphorase.

Histochemistry. The brain and spinal cord sections were rinsed in phosphate buffer then floated in a diluent containing $0.3 \%$ Triton-X 100 in phosphate buffer while being shaken for 1-2 min. The staining was then performed by incubating the tissue in a solution containing equal parts of nitro-blue tetrazolium chloride $(0.4 \mathrm{mg} / \mathrm{ml}$ in phosphate buffer) and NADPH ( $1 \mathrm{mg} / \mathrm{ml}$ in buffer) for about $20-24 \mathrm{~h}$ at $37^{\circ} \mathrm{C}$. The sections were rinsed twice in phosphate buffer solution and mounted using glycerol gel (Weinberg et al. 1996).

Gallocyanin chrom alum stain. Brain and spinal cord sections were stained with a mixture of gallocyanin and chromium potassium sulphate (chrom alum) overnight. The sections then were rinsed in running tap water, dehydrated using ascending grades of alcohol and cleared in xylene and mounted using DPX (Einarson 1932).

Image analysis. Six brain and spinal cord sections from each animal, cut at $100 \mu \mathrm{m}$ apart, were processed for NADPH-diaphorase histochemistry and analyzed morphometrically using computerized image analysis. The image analyzer consisted of a colored video camera, colored monitor, hard disc of IBM personal computer connected to the microscope, and controlled by Leica Qwin 500 software (England). The image analyzer was first calibrated automatically to convert the measurement units (pixels) produced by the image analyzer program into actual micrometer units. The area and area percentage of NADPH-diaphorase stained sections of the rat brain and spinal cord were measured using an objective lens of magnification 10. Using the color detect, NADPH-diaphorase histochemical reaction was masked by a blue binary color. The area and area percentage was calculated in relation to a standard measuring frame.

\section{Statistical analysis}

All values are expressed as means \pm SD (standard deviation). Comparison between the two age groups was done using unpaired Student $t$-test. Comparison within the same group was done using paired-t-test; $p<0.05$ was considered significant. 


\section{Results}

\section{Effect of aging on basal pain threshold}

Figure 1 shows that there is a significant increase in pain threshold in aged rats ( 24 months) when compared with young rats $(2$ months $)(p<0.01)$ by using both mechanical and chemical stimuli.

Effect of systemic administration of L-arginine and L-NAME on paw pressure threshold

Figure 2 shows a significant decrease of paw pressure threshold in aged rats only after systemic administration of L-arginine when compared with basal value $(p<0.01)$. Pain threshold is decreased in aged rats by $35.06 \%$ but increased in young rats by $9.49 \%$ after systemic L-arginine administra-
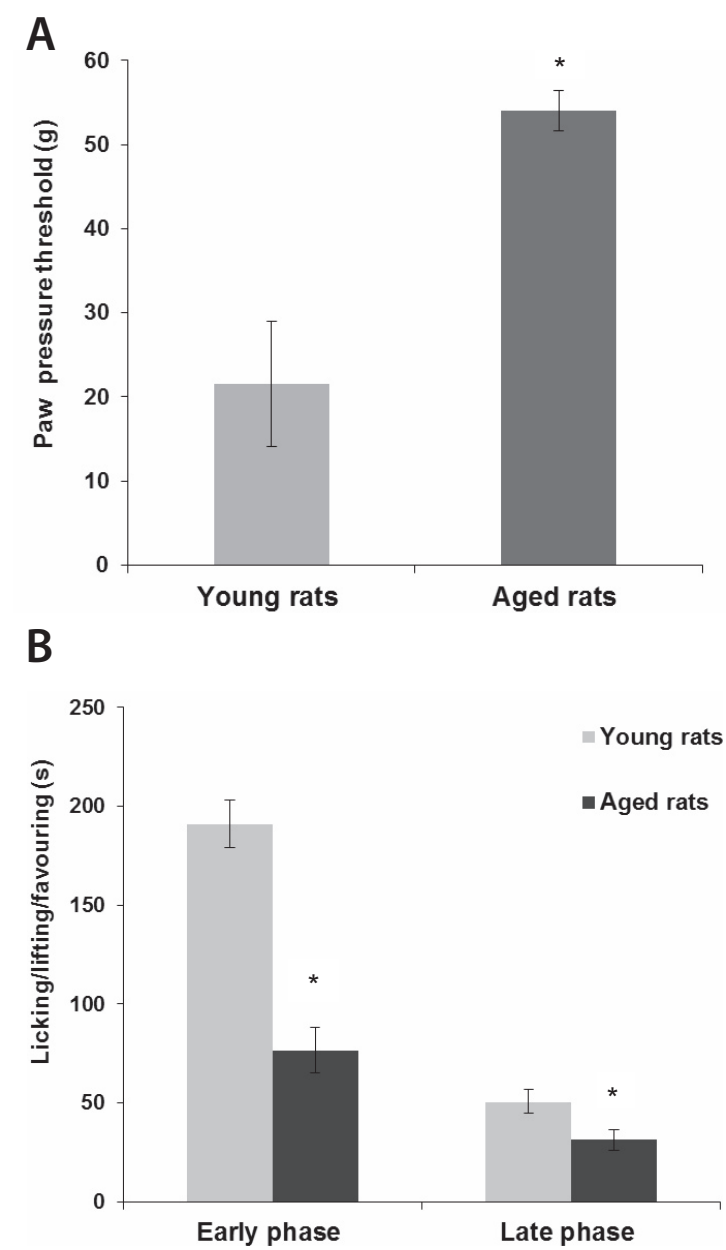

Figure 1. Basal pain threshold in young ( 2 month-old, $n=12$ ) and aged (24 month-old, $n=12$ ) rats in response to paw pressure test (A) and formalin injection test (B). Values are expressed as mean \pm SD. ${ }^{*} p \leq 0.01 v s$. young rats (Student's unpaired $t$-test).

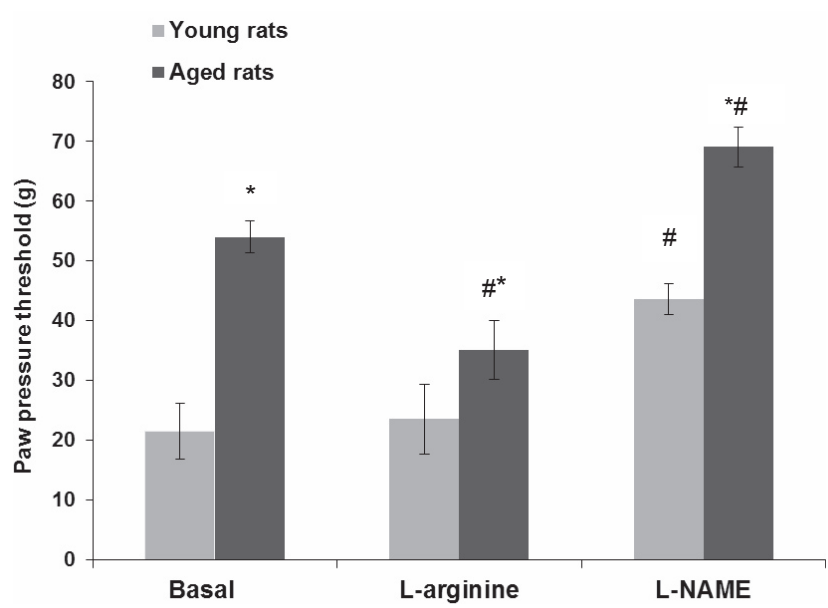

Figure 2. Effect of systemic adminstration of L-arginine $(200 \mathrm{mg} / \mathrm{kg})$ and L-NAME $(50 \mathrm{mg} / \mathrm{kg})$ on paw pressure threshold in young ( 2 month-old) and aged (24 month-old) rats. Values are expressed as mean \pm SD. ${ }^{*} p<0.01 v$ s. young rats (unpaired $t$-test). $\# p<0.01$ $v s$. basal value (paired $t$-test).

tion. On the other hand, systemic administration of L-NAME caused significant increase in pain threshold in both young and aged rats $(p<0.01)$, but, the effect was more pronounced in young $(106.48 \%)$ that in aged rats $(21.80 \%)$.

Effect of i.c.v. injection of L-arginine and L-NAME on paw pressure threshold

Figure 3 shows i.c.v. administration of L-arginine caused non significant decrease of paw pressure threshold in young rats by $26.07 \%$ and in aged rats by $9.29 \%$ when compared

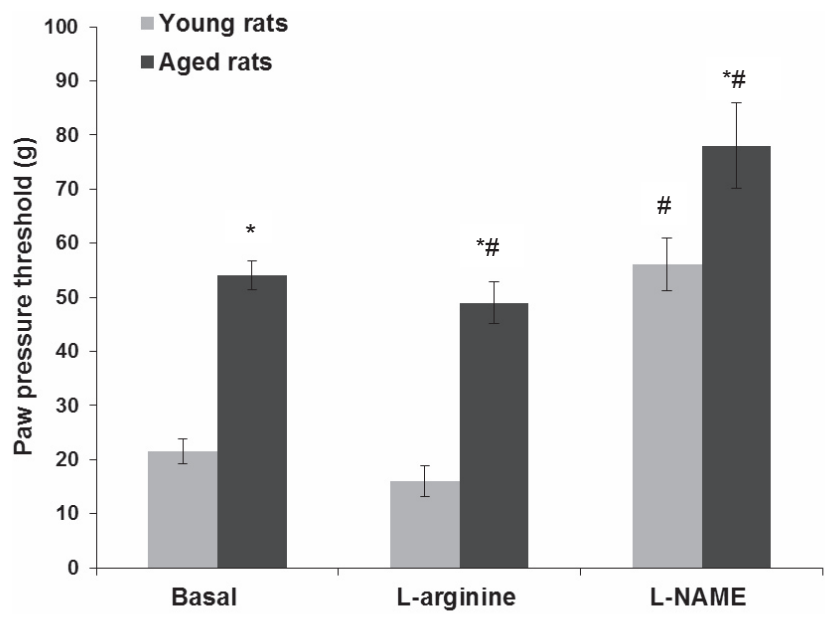

Figure 3. Effect of i.c.v. adminstration of $\mathrm{L}$-arginine $(1 \mu \mathrm{g})$ and L-NAME $(1 \mu \mathrm{g})$ on paw pressure threshold in young and aged rats. Values are expressed as mean $\pm \mathrm{SD}$. ${ }^{\star} p<0.01 v s$. young rats (unpaired $t$-test). $\# p<0.01 v$ s. basal value (paired $t$-test). 
with basal value. However, i.c.v. administration of L-NAME caused significant increase in pain threshold in both young rats (by $160.84 \%$ ) and aged rats (by $44.39 \%$ ) when compared with basal value $(p<0.01)$.

Effect of systemic administration of L-arginine and L-NAME on formalin-induced pain threshold

Figure 4A shows non-significant increase in formalin-induced pain threshold during early phase in both young (by $8.23 \%$ ) and aged (by $18.16 \%$ ) rats after systemic administration of Larginine when compared with basal value. On the other hand, systemic administration of L-NAME caused significant decrease of pain threshold during early phase in both young (by 41.9\%) and aged (by $57 \%)$ rats $(p<0.01)$. In late phase, L-NAME caused significant decrease of pain threshold in young (by $32.53 \%$ ) and aged (by $36.42 \%)$ rats $(p<0.01)$, while, L-arginine caused no significant change in both young and aged rats (Fig. 4B).

Effect of i.c.v. administration of L-arginine and L-NAME on formalin-induced pain threshold

Figure 5A shows significant increase in formalin-induced pain threshold during early phase in both young (by $28.53 \%$ ) and aged (by $79.48 \%$ ) rats after i.c.v. administration of L-arginine when compared with basal value $(p<0.001)$. However, i.c.v. administration of L-NAME caused significant decrease of pain threshold during early phase in both young (by $47.25 \%$ ) and aged (by $37.91 \%)$ rats $(p<0.01)$. In late phase, i.c.v. administration of L-arginine caused significant increase in pain threshold in young (by 192.81\%) and aged rats (by 66.45\%) $(p<0.001$; Fig. 5A). Unexpectly, i.c.v. administration of L-NAME caused significant increase in formalin-induced pain threshold during late phase in both young $(30.76 \%)$ and aged $(49.84 \%)$ rats when compared with basal value $(p<0.001$; Fig. 5B).

\section{NADPH-diaphorase activity}

Quantitative evaluation of NADPH-diaphorase activity by image analysis revealed significant increase in the mean area and area \% of the NADPH-diaphorase histochemical reaction in brain and spinal cord sections of young rats in comparison to that of aged rats (Table 1).

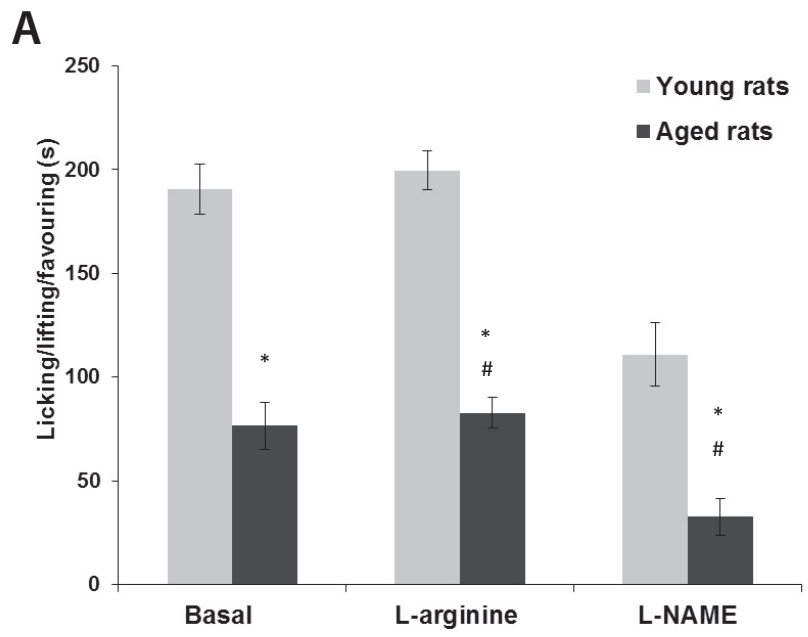

B

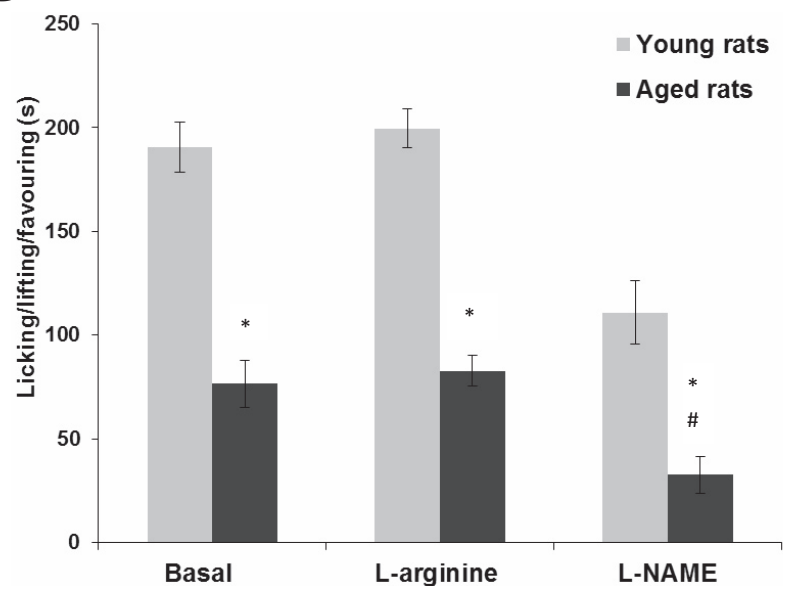

Figure 4. Effect of systemic L-arginine $(200 \mathrm{mg} / \mathrm{kg})$ and L-NAME $(50 \mathrm{mg} / \mathrm{kg})$ adminstration on time of paw licking and lifting (s) induced by formalin injection in young ( 2 month-old) and aged (24 month-old) rats during early phase (A) and late phase (B). Values are expressed as mean $\pm \mathrm{SD} .{ }^{*} p<0.01 v s$. young rats (unpaired $t$-test). $\# p<0.01 v$ s. basal value (paired $t$-test).

Examination of the CA1 region of the hippocampus showed increase in the activity of NOS of the young rats (Fig. 6b) in comparison to the aged rats (Fig. 6e). Moreover,

Table 1. Descriptive statistics of the mean area and area \% of the NADPH-diaphorase histochemical reaction of the brain and spinal cord sections

\begin{tabular}{lcccc}
\hline & \multicolumn{2}{c}{ Young rats } & & \multicolumn{2}{c}{ Aged rats } \\
\cline { 2 - 3 } \cline { 5 - 5 } & Mean area $\left(\mu \mathrm{m}^{2}\right)$ & Mean area $\%$ & & Mean $\operatorname{area}^{2}\left(\mu \mathrm{m}^{2}\right)$ \\
\hline CA1 region & $103612.26 \pm 1892.41^{\star}$ & $2.61 \pm 0.05^{*}$ & & $16179.19 \pm 331.27$ \\
Choroid plexus & $72506.44 \pm 5348.76^{*}$ & $1.83 \pm 0.13^{*}$ & & $38915.33 \pm 1817.58$ \\
Lumbosacral region & $624811.57 \pm 63586.72^{*}$ & $15.75 \pm 1.6^{*}$ & & $41068.47 \pm 3140.54$ \\
\hline
\end{tabular}

${ }^{*} p<0.05$ is statistically significant $v s$. old rats (unpaired Student $t$-test). Data are expressed as mean \pm SD. 
A

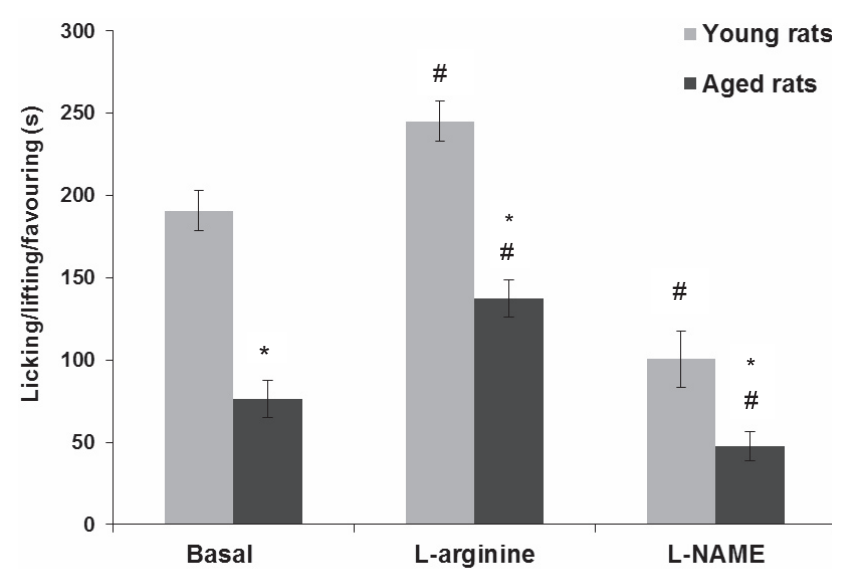

B

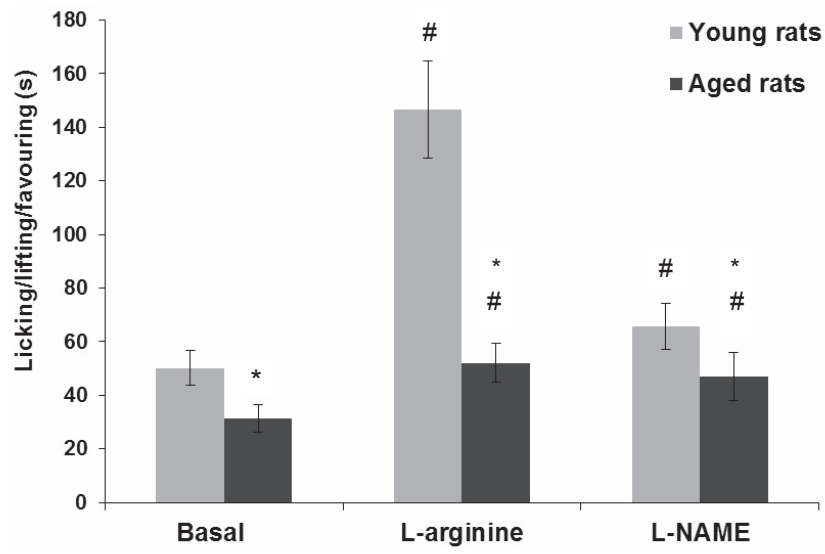

Figure 5. Effect of i.c.v. adminstration of L-arginine $(1 \mu \mathrm{g})$ and L-NAME $(1 \mu \mathrm{g})$ on time of paw licking and lifting induced by formalin injection in young ( 2 month-old) and aged (24 month-old) rats during early phase (A) and late phase (B). Values are expressed as mean \pm SD. ${ }^{\star} p<0.01 v s$. young rats (unpaired $t$-test). $\# p<0.01 v$ s. basal value (paired $t$-test).
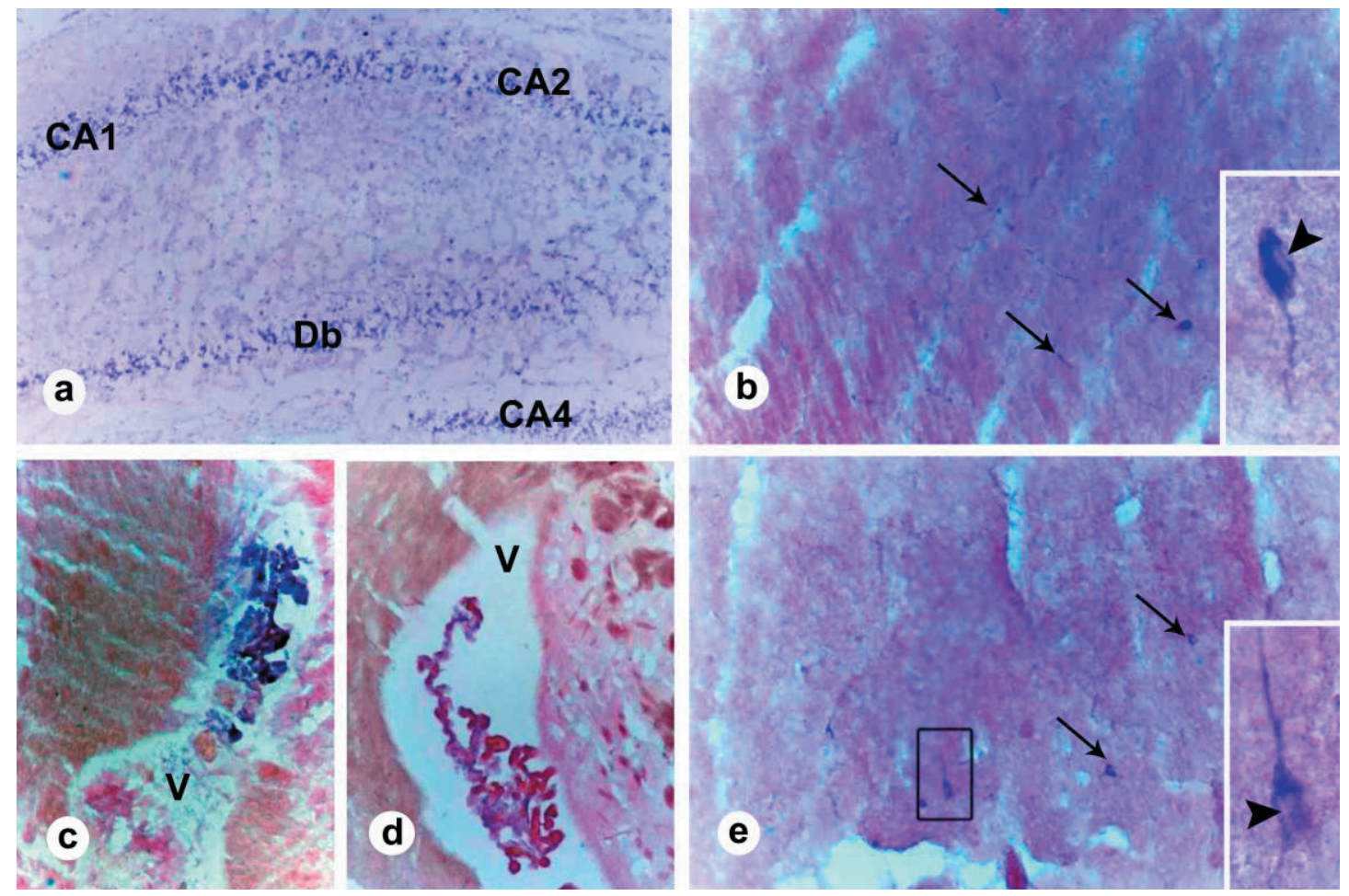

Figure 6. Photomicrographs of the rat brain. Section of the hippocampus of adult rat (a) showing its different regions: the CA1, CA2 and CA4 fields of cornu ammonis and the buried (Db) blade of the dentate gyrus (Gallocyanin chrom alum stain, magnification $\times 40$ ). Section of the hippocampus at CA1 area of young rat (b) showing positive NADPH-diaphorase cells (arrows). The inset shows a higher magnification of one of these cells. Note the unstained nucleus (arrowhead) (NADPH-diaphorase histochemistry, $\times 100$; inset, $\times 400$ ). Section of the brain of the ventricle of young rat (c) and aged rat (d), showing positively stained NADPH-diaphorase endothelial cells lining the choroid plexus (NADPH-diaphorase histochemistry, $\times 40$ ). Section of the hippocampus at CA1 area of young rat (e) showing few positive NADPH-diaphorase cells (arrows). The inset shows a higher magnification of one of these cells (rectangle). Note the unstained nucleus (arrowhead) (NADPH-diaphorase histochemistry, $\times 100$; inset, $\times 400$ ). 
NADPH-diaphorase reaction product was also localized to the vascular endothelial lining and choroidal epithelial cells of both young and aged rats but the reaction was more intense in the young rats (Fig. $6 \mathrm{c}$ and $\mathrm{d}$ ).

The NOS activity was examined in the coronal sections of the lumbar segments of the spinal cord of the rats and it was found that the number of NADPH-diaphorase cells was greater in the dorsal horn (lamina I-IV) in the young rats in comparison to that of the aged rats (Fig. $7 \mathrm{a}$ and $\mathrm{b}$ ). On the other hand, few positive cells were found around the central canal (lamina $\mathrm{X}$ ) in the aged rats (Fig. $7 \mathrm{c}$ and d).

\section{Discussion}

The effect of age on pain sensitivity is still unclear, and its underlying mechanisms are poorly understood. Moreover, the precise role of NO in pain is also uncertain as studies have indicated both pronociceptive and antinociceptive roles for NO. Thus, our study aimed to examine the role of $\mathrm{NO}$ in pain sensitivity in aged rats, using 2 tests of nociception - formalin and paw pressure tests. In paw pressure test an increasing force $(16 \mathrm{~g} / \mathrm{s})$ was applied to the right hind paw and the pressure that forced the rat to withdraw the paw is taken as an index of nociceptive mechanical withdrawal threshold. The pressure applied to the hind limb activates certain slowly adapting mechanoreceptors that send signals via C fibers to spinal cord (Lewin and Moshourab 2004). On the other hand, the nociceptive response to formalin consists of 2 phases; an early phase ( $0-5 \mathrm{~min})$ which is considered to be produced by a direct activation of peripheral afferent $\mathrm{C}$ fibers by formalin. The second late phase of the response (15-60 $\mathrm{min}$ ) is mediated by ongoing stimulation of nociceptors by inflammatory mediators from injured tissues and/or central sensitization as a response to first early phase (Tang et al. 2007). The use of 2 different nociceptive stimuli and the fact that formalin test with its 2 phases of nociception is reflecting different pathophysiological processes was helpful to augment the outcome of the experiments.

The results of the present study show that the nociceptive threshold of aged rats (24 month-old) is significantly higher than that of young animals ( 2 month-old) to both mechanical and chemical stimuli, indicating a decrease of the reactivity to pain in aged rats. This finding of the decreased pain sensitivity to mechanical nociceptive stimuli is
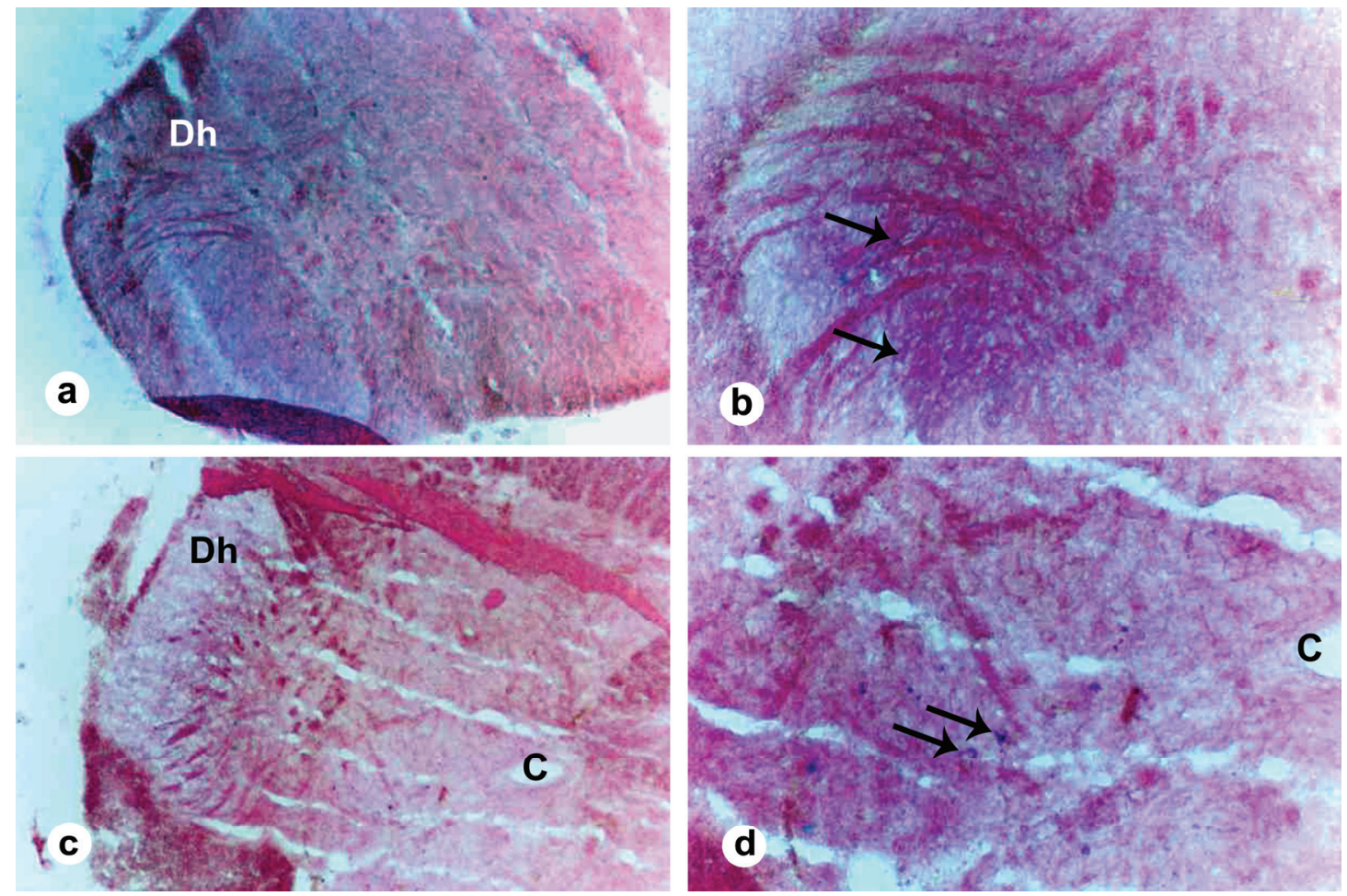

Figure 7. Photomicrographs of the rat spinal cord. Section of the spinal cord of young rat (a) and of aged rat (c), showing the dorsal horn (Dh) and the central canal (C). Note the increase in the intensity of the staining in lamina (I-IV) of the dorsal horn of young rat in comparison to aged rat (NADPH-diaphorase histochemistry, $\times 40$ ). b. Higher magnification of (a) showing positive NADPH-diaphorase cells (arrows) in the dorsal horn (NADPH-diaphorase histochemistry, $\times 100)$. d. Higher magnification of (c) showing positive NADPHdiaphorase cells (arrows) in the grey matter surrounding the central canal (NADPH-diaphorase histochemistry, $\times 100$ ). 
in accordance with the study of Akunne and Soliman (1994) who found less sensitivity of 24 month-old Fischer-344 rats to paw pressure test when compared with 3 month-old rats. By contrast, Jourdan et al. (2000) have shown a decreased mechanical withdrawal threshold in response to paw pressure test in 24 month-old Lou/c rats than in middle aged and mature rats. On the other hand, the present finding of the increase in pain threshold induced by formalin in aged rats is different from the study by Gagliese and Melzacka (1999) who showed that there was no difference between 3 and 24 month-old Long-Evans rats. A recent study by Hagiwara et al. (2010) has found hypersensitivity to formalin injection in middle aged (11 month-old) female Sprague Dawley rats.

One important aim of the present study was to further elucidate the role of NO in nociception. In response to pressure nociceptive stimulus, the present results shows that with either i.p. or i.c.v. administration of L-NAME, the sensitivity to pain decreased in both the young and aged rats suggesting a pronociceptive role of NO that is mediated both peripherally and centrally. Further, this antinociceptive effect of L-NAME was more pronounced in the young rats than in the aged rats suggesting that the pronociceptive role of $\mathrm{NO}$ in response to paw pressure is decreasing with age. Moreover, the decrease of pain sensitivity in the young rats was more marked with i.c.v. administration than with i.p. L-NAME administration (160\%) that may reflect a greater central role of NO in pain perception in the young rats than in the aged ones. Indeed, the results of the histochemistry study revealed a greater activity of NADPH-diaphorase cells in hippocampus sections from young rats relative to aged rats indicating greater activity of neuronal NO; a finding that is consistent with the above assumption that the central pronociceptive role of $\mathrm{NO}$ is decreasing with age. There is evidence from previous studies about a possible role of hippocampus CA1 region in nociception (Khanna 1997; Soleimannejad et al. 2007) and that is most probably through a mechanism related to NOS activation (Hashemi et al. 2010). Thus, the study extends this to its involvement in blunted pain sensitivity in aged rats.

On the other hand, using the NO precursor L-arginine, the sensitivity to pressure-induced pain increased after systemic administration in the aged rats only, while there was no change in the pain reactivity after i.c.v. L-arginine in both groups (see Fig. 2 and 3). This finding suggests that with augmenting the synthesis of $\mathrm{NO}$ with L-arginine, the pronociceptive effect of $\mathrm{NO}$ is mediated peripherally in the aged rats only while it has no central role in nociception in both groups. The fact that the aged rats showed less reactivity to paw pressure when compared with the young rats (Fig. 1) and that their reactivity to pain was enhanced by systemic L-arginine supports again the above suggestion that the pronociceptive role of NO in response to paw pressure decreases with age. What is interesting here is that i.c.v. L-arginine had no effect on pain reactivity in both groups. This might be related to the dose used in the present experiments as in the study by Sousa and Prado (2001); higher dose of the NO donor (3-morpholinosydnonimine (SIN-1)) had no effect or increased the pain produced by chronic ligature of sciatic nerve, but had antinociceptive effect in rat tail-flick test. The present findings regarding the role of NO in nociception induced by mechanical stimuli are in agreement with previous studies that used also the non selective NOS inhibitor L-NAME although they have used different mechanical stimuli as tail flick test (Iwamoto and Marion 1994) or von Frey hair test (Hao and Xu 1996; Sousa and Prado 2001). However, studies using other NO inhibitors have not produced consistent results as intraplantar or i.c.v. L-NOARG (Aley et al. 1998; Xu et al. 1995) and intrathecal 7-nitroindazole (7-NI) (Machelska et al. 1997).

Regarding the response to formalin injection, i.p. L-arginine did not affect the pain threshold in both age groups either during the acute or the tonic phase (Fig. 4a and b). By contrast, i.c.v. administration of L-arginine enhanced pain sensitivity in both groups during acute and tonic phases of the nociceptive response, but with greater influence in the young rats during the late phase. This suggests a central pronociceptive role of L-arginine-NO pathway in response to formalin that is more evident for the late chronic phase in the young rats, indicating that $\mathrm{L}$-arginine-NO pronociceptive pathway is decreasing with age. What is unexplained here why i.c.v. L-arginine did not change the nociceptive response to paw pressure while enhanced it in response to formalin; whether this is related to the dose or it is related to the modality of pain tested warrants further investigation.

On the other hand, i.p. L-NAME inhibited the acute and tonic nociceptive response to formalin injection in both groups of rats as indicated by the shorter time spent licking or lifting the paw, with the young rats still more sensitive to pain than the aged animals. This suggests a pronociceptive role of $\mathrm{NO}$ that is mediated peripherally. However, with i.c.v. L-NAME, the decrease of pain reactivity was noticed only during early phase while the sensitivity to pain increased during late phase indicating a dual role of $\mathrm{NO}$ with central inhibition of its synthesis; exerting a pronociceptive effect in response to acute pain and an antinociceptive effect during the tonic or chronic phase of pain. Indeed, previous studies on formalin-induced nociceptive response after NOS inhibitors have shown a complex role of NO. Thus, Malmberg and Yaksh (1993) and Sakurada et al. (1996) reported that i.p., intrathecal or i.c.v. L-NAME inhibited the nociceptive response during the late phase with little or no effect on the acute phase. However, other studies have reported that intrathecal injection of L-NAME greatly reduced the acute phase (Sakurada et al. 2001) and the tonic phase of nociceptive response to formalin (Meller et al. 1994). Recently, Kolesnikov et al. (2004) demonstrated that local application of the NOS inhibitor, NOArg, did not affect either phase of the formalin test, while supraspinal blockade of nNOS with 
NOArg reduced both phases, suggesting the presence of opposing nNOS systems in pain modulation.

In conclusion, the present experiments show that the pain response evoked by either paw pressure or formalin injection is decreased (blunted) in aged Sprague-Dawley rats as compared to young animals. It seems that augmenting the synthesis of peripheral NO with L-arginine has a pronociceptive effect on response to paw pressure in aged rats only, while central L-arginine enhances pain response induced by formalin in both groups. Further, either peripheral or central inhibition of NO synthase with L-NAME has antinociceptive effect on pain response induced by paw pressure and during the acute phase induced by formalin injection in both groups. This antinociceptive effect of L-NAME seems to be more manifest in the young rats than in the aged rats. On the other hand, L-NAME (i.c.v.) enhanced pain response evoked during the late phase of formalin test in both groups, suggesting that centrally synthesized NO has an antinociceptive effect during the chronic stage of pain evoked by formalin.

\section{References}

Abacioğlu N., Ozmen R., Cakici I., Tunçtan B., Kanzik I. (2001): Role of L-arginine/nitric oxide pathway in the antinociceptive activities of morphine and mepyramine in mice. Arzneimittelforschung 51, 977-983

Akunne H. C., Soliman K. F. A. (1994): Serotonin modulation of pain responsiveness in the aged rat. Pharmacol. Biochem. Behav. 48, 411-416 http://dx.doi.org/10.1016/0091-3057(94)90545-2

Aley K. O., McCarter G., Levine J. D. (1998): Nitric oxide signaling in pain and nociceptor sensitization in the rat. J. Neuroscience 18, 7008-7017

Brito G. A., Sachs D., Cunha F. Q., Vale M. L., Lotufo C. M., Ferreira S. H., Ribeiro R. A. (2006): Peripheral antinociceptive effect of pertussis toxin: activation of the arginine/NO/cGMP/PKG/ ATPsensitive K channel pathway. Eur. J. Neurosci. 24, 1175-1181 http://dx.doi.org/10.1111/j.1460-9568.2006.04991.x

Chen S. R., Pan H. L. (2003): Spinal nitric oxide contributes to the analgesic effect of intrathecal (D-Pen2, D-Pen5)-enkephalin in normal and diabetic rats. Anesthesiology 98, 217-222 http://dx.doi.org/10.1097/00000542-200301000-00033

Crisp T., Stafinsky J. L., Hoskins D. L., Dayal B., Chinrock K. M., Uram M. (1994): Effects of aging on spinal opioid induced antinociception. Neurobiol. Aging 15, 169-174 http://dx.doi.org/10.1016/0197-4580(94)90108-2

Crosby S. J., Knapp C. M, Kornetsky C. (2006): Nociceptive threshold and analgesic response to morphine in aged and young adult rats as determined by thermal radiation and intracerebral electrical stimulation. Pharmacol. Biochem. Behav. 84, 148-157 http://dx.doi.org/10.1016/j.pbb.2006.04.021

Duarte I. D., Ferreira S. H. (1992): The molecular mechanism of central analgesia induced by morphine or carbachol and the L-argininenitric oxide-cGMP pathway. Eur. J. Pharmacol. 221, 171-174 http://dx.doi.org/10.1016/0014-2999(92)90789-7
Duarte I. D., Ferreira S. H. (2000): L-NAME causes antinociception by stimulation of the arginine-NO-cGMP pathway. Mediators Inflamm. 9, 25-30 http://dx.doi.org/10.1080/09629350050024348

Einarson L. (1932): A method for progressive selective staining of nissle and nuclear substance in nerve cells. Am. J. Pathol. 8, 295-308

England S., Bevan S., Docherty R. J. (1996): PGE2 modulates the tetrodotoxin-resistant sodium current in neonatal rat dorsal root ganglion neurones via the cyclic AMP-protein kinase A cascade. J. Physiol. (Lond) 495, 429-440

Freire M. M., Guimarães J. S., Gomes-Leal W., Pereira A. (2009): Pain modulation by nitric oxide in the spinal cord. Front. Neurosci. 3, 175-181 http://dx.doi.org/10.3389/neuro.01.024.2009

Gagliese L., Melzack R. (1999): Age differences in the response to the formalin test in rats. Neurobiol. Aging 20, 699-707 http://dx.doi.org/10.1016/S0197-4580(99)00061-5

Garthwaite J. (2008). Concepts of neural nitric oxide-mediated transmission. Eur. J. Neurosci. 27, 2783-2802 http://dx.doi.org/10.1111/j.1460-9568.2008.06285.x

Gholipour T., Jabbarzadeh A., Riazi K., Rasouli A., Nezami B. G., Sharifzadeh M., Dehpour A. R. (2008): Role of nitric oxide in the anticonvulsive effect of progesterone. Epilepsy Behav. 13, 579-584 http://dx.doi.org/10.1016/j.yebeh.2008.07.011

Goicoechea C., Ormazabal M. J., Alfaro M. J., Martin M. I. (1997): Age related changes in nociception behavior and monoamine levels in rats. Gen. Pharm. 28, 331-336 http://dx.doi.org/10.1016/S0306-3623(96)00222-4

Haghikia A., Mergia E., Friebe A., Eysel U. T., Koesling D., Mittmann T. (2007): Long-term potentiation in the visual cortex requires both nitric oxide receptor guanylyl cyclases. J. Neurosci. 27, 818-823 http://dx.doi.org/10.1523/JNEUROSCI.4706-06.2007

Hagiwara H., Kimura F., Mitsushima D., Funabashi T. (2010): Formalin-induced nociceptive behavior and c-Fos expression in middle-aged female rats. Physiol. Behav. 100, 101-104 http://dx.doi.org/10.1016/j.physbeh.2010.02.004

Haley T. J., Mc Cormick W. G. (1957): Pharmacological effects produced by intracerebral injections of drugs in the conscious mouse. Br. J. Pharmacol. 12, 12-15

Handy R. L., Moore P. K. (1998): Effects of selective inhibitors of neuronal nitric oxide synthase on carrageenan-induced mechanical and thermal hyperalgesia. Neuropharmacology 37, 37-43 http://dx.doi.org/10.1016/S0028-3908(97)00201-3

Hao J. X., Xu X. J. (1996): Treatment of a chronic allodynia-like response in spinally injured rats: effects of systemically administered excitatory amino acid receptor antagonists. Pain 66, 279-285 http://dx.doi.org/10.1016/0304-3959(96)03019-9

Harkins S. W., Price D. D., Bush F. M., Small R. E. (1994): Geriatric pain. In: Textbook of Pain. (Eds. P. D. Wall and M. Melzack), pp 769-784, 3rd ed, Churchill Livingstone, New York

Hashemi M., Karami M., Zarrindast M. R., Sahebgharani M. (2010): Role of nitric oxide in the rat hippocampal CA1 in morphine antinociception. Brain Res. 1313, 79-88 http://dx.doi.org/10.1016/j.brainres.2009.11.020

Holthusen H., Arndt J. O. (1994): Nitric oxide evokes pain in humans on intracutaneous injection. Neurosci. Lett. 165, 71-74 http://dx.doi.org/10.1016/0304-3940(94)90712-9 
Holthusen H., Arndt J. O. (1995): Nitric oxide evokes pain at nociceptors of the paravascular tissue and veins in humans. J. Physiol. (Lond) 487, 253-258

Hopper R. A., Garthwaite J. (2006): Tonic and phasic nitric oxide signals in hippocampal long-term potentiation. J. Neurosci. 26, 11513-11521 http://dx.doi.org/10.1523/JNEUROSCI.2259-06.2006

Islam A. K., Cooper M. L., Bodnar R. J. (1993): Interactions among aging, sex and gonadectomy effects upon morphine antinociception in rats. Physiol. Behav. 54, 45-53 http://dx.doi.org/10.1016/0031-9384(93)90042-E

Iwamoto E. T., Marion L. (1994): Pharmacological evidence that nitric oxide mediates the antinociception produced by muscarinic agonists in the rostral ventral medulla of rats. J. Pharmacol. Exp. Ther. 269, 699-708

Jourdan D., Boghossian S., Alloui A., Veyrat-Durebex C., Coudore M. A., Eschalier A., Alliot J. (2000): Age-related changes in nociception and effect of morphine in the Lou rat. European J. Pain 4, 291-300 http://dx.doi.org/10.1053/eujp.2000.0188

Khanna S. (1997): Dorsal hippocampus field CA1 pyramidal cell responses to a persistent versus an acute nociceptive stimulus and their septal modulation. Neuroscience 77, 713-721 http://dx.doi.org/10.1016/S0306-4522(96)00456-3

Kolesnikov Y., Cristea M., Oksman G., Torosjan A., Wilson R. (2004): Evaluation of the tail formalin test in mice as a new model to assess local analgesic effects. Brain Res. 1029, 217-223 http://dx.doi.org/10.1016/j.brainres.2004.09.058

Kutlu S., Canpolat S., Sandal S., Ozcan M., Sarsilmaz M., Kelestimur H. (2003): Effects of central and peripheral administration of leptin on pain threshold in rats and mice. Neuro. Endocrinol. Lett. 24, 193-196

Lewin G. R., Moshourab R. (2004): Mechanosensation and pain. J. Neurobiol. 61, 30-44 http://dx.doi.org/10.1002/neu.20078

Machelska H., Labuz D., Przewlocki R. Przewlocki B. (1997): Inhibition of nitric oxide synthase enhances antinociception mediated by $\mathrm{Mu}$, Delta and Kappa opioid receptors in acute and prolonged pain in the rat spinal cord. J. Pharmacol. Exp. Ther. 282, 977-984

Malmberg A. B., Yaksh T. L. (1993): Spinal nitric oxide synthesis inhibition blocks NMDA-induced thermal hyperalgesia and produces antinociception in the formalin test in rats. Pain 54, 291-300 http://dx.doi.org/10.1016/0304-3959(93)90028-N

Meller S. T., Cummings C. P., Traub R. J., Gebhart G. F. (1994): The role of nitric oxide in the development and maintenance of the hyperalgesia produced by intraplantar injection of carrageenan in the rat. Neuroscience 60, 367-374 http://dx.doi.org/10.1016/0306-4522(94)90250-X

Milligan E. D., Watkins L. R. (2009): Pathological and protective roles of glia in chronic pain. Nat. Rev. Neurosci. 10, 23-36 http://dx.doi.org/10.1038/nrn2533

Moncada S. (1994): Nitric Oxide (Review). J. Hypertens. (Suppl. 12), S35-39

Moore P. K., Oluyomi A. O., Babbedge R. C., Wallace P., Hart S. L. (1991): L-NG-nitro arginine methyl ester exhibits antinociceptive activity in the mouse. Br. J. Pharmacol. 102, 198-202
Pataki I., Telegdy G. (1998): Further evidence that nitric oxide modifies acute and chronic morphine actions in mice. Eur. J. Pharmacol. 357, 157-162 http://dx.doi.org/10.1016/S0014-2999(98)00561-5

Patil C. S., Jain N. K., Singh V. P., Kulkarni S. K. (2004): CholinergicNO-cGMP mediation of sildenafil-induced antinociception. Indian J. Exp. Biol. 42, 361-367

Przesmycki K., Dzieciuch J. A., Czuczwar S. J., Kleinrok Z. (1999): Nitric oxide modulates spinal antinociceptive effect of clonidine but not that of baclofen in the formalin test in rats. Eur. Neuropsychopharmacol. 9,115-121 http://dx.doi.org/10.1016/S0924-977X(98)00014-5

Randall L. O., Sellito J. J. (1957): A method for measurement of analgesic activity on inflamed tissue. Arch. Int. Pharmacodyn. 111, 409-419

Sakurada T., Sugiyama A., Sakurada C., Tan-No K., Yonezawa A., Sakurada S., Kisara K. (1996): Effect of spinal nitric oxide inhibition on capsaicin-induced nociceptive response. Life Sci. 59, 921-930 http://dx.doi.org/10.1016/0024-3205(96)00390-6

Sakurada C., Sugiyama A., Nakayama M., Yonezawa A., Sakurada S., Tan-No K., Kisara K, Sakurada T. (2001): Antinociceptive effect of spinally injected L-NAME on the acute nociceptive response induced by low concentrations of formalin. Neurochem. Int. 38, 417-423

http://dx.doi.org/10.1016/S0197-0186(00)00110-8

Soleimannejad E., Naghdi N., Semnanian S., Fathollahi Y., Kazemnejad A. (2007): Antinociceptive effect of intra-hippocampal CA1 and dentate gyrus injection of MK801 and AP5 in the formalin test in adult male rats. Eur. J. Pharmacol. 562, 39-46 http://dx.doi.org/10.1016/j.ejphar.2006.11.051

Sousa A. M., Prado W. A. (2001): The dual effect of a nitric oxide donor in nociception. Brain Res. 897, 9-19 http://dx.doi.org/10.1016/S0006-8993(01)01995-3

Taiwo Y. O., Levine J. D. (1989): Prostaglandin effects after elimination of indirect hyperalgesic mechanisms in the skin of the rat. Brain Res. 492, 397-399 http://dx.doi.org/10.1016/0006-8993(89)90928-1

Tang L., Chen Y., Chen Z., Blumberg P. M., Kozikowski A. P., Wang Z. J. (2007): Antinociceptive pharmacology of N-(4-chlorobenzyl)-N'-(4-hydroxy-3-iodo-5-methoxybenzyl) thiourea, a highaffinity competitive antagonist of the transient receptor potential vanilloid 1 receptor. J. Pharmacol. Exp. Ther. 321, 791-798 http://dx.doi.org/10.1124/jpet.106.117572

Wall P. D., Woolf C. J. (1984): Muscle but not cutaneous C-afferent input produces prolonged increases in the excitability of the flexion reflex in the rat. J. Physiol. 356, 443-458

Weinberg R. J., Valtschanoff J. G., Schmidt H. H. H. W. (1996): The NADPH Diaphorase histochemical staining. In: Methods in Nitric Oxide Research. (Eds. M. Ferelisch, J. Stamler), pp. 237-248, John Wiley \& sons Ltd., London

Xu J. Y., Pieper G. M., Tseng L. F. (1995): Activation of a NO-cyclic GMP system by NO donors potentiates beta-endorphin-induced antinociception in the mouse. Pain 63, 377-383 http://dx.doi.org/10.1016/0304-3959(95)00067-4

Received: August 30, 2011

Final version accepted: January 9, 2012 